\title{
Neoadjuvant chemoradiotherapy for locally advanced thymic tumors: A phase II, multi-institutional clinical trial
}

\author{
Robert J. Korst, MD, ${ }^{\mathrm{a}, \mathrm{b}, \mathrm{c}}$ Andrea Bezjak, MD, ${ }^{\mathrm{c}, \mathrm{d}}$ Shanda Blackmon, MD, ${ }^{\mathrm{e}}$ Noah Choi, MD ${ }^{\mathrm{f}}$ \\ Panos Fidias, MD, ${ }^{\mathrm{g}}$ Geoffrey Liu, MD, ${ }^{\mathrm{c}, \mathrm{h}}$ Alexander Marx, MD, ${ }^{\mathrm{c}, \mathrm{i}}$ Cameron Wright, MD, ${ }^{\mathrm{c}, \mathrm{j}}$ \\ Susan Mock, RN, ${ }^{a}$ John R. Rutledge, MAS, ${ }^{a}$ and Shaf Keshavjee, MD $^{\mathrm{c}, \mathrm{k}}$
}

Objective: To determine the response rate, toxicity, and rate of complete resection after induction chemoradiotherapy for locally advanced thymic tumors, which were defined by specific radiographic criteria.

\begin{abstract}
Methods: A single-arm, pilot trial was conducted at 4 institutions. Patients with thymoma or thymic carcinoma who met specific criteria on computed tomography were accrued. Induction therapy consisted of 2 cycles of cisplatin and etoposide combined with 45 Gy of thoracic radiotherapy. Patients underwent computed tomography and positron emission tomography before and after induction therapy and then resection was attempted. Postoperative chemoradiotherapy was administered in selected patients. The primary endpoint was the pathologic response to induction therapy. The secondary endpoints were toxicity, surgical complications, radiographic response, and the rate of $\mathrm{R} 0$ resection.
\end{abstract}

Results: A total of 22 patients were accrued during a 5-year period (1 patient withdrew before starting induction therapy). Of the 22 patients, 21 completed induction therapy, and $9(41 \%)$ experienced grade 3 or 4 toxicity. A total of 10 patients had a partial radiographic response and 11 had stable disease. Of the 21 patients, 17 (77\%) underwent an R0 resection, $3(14 \%)$ an R1 resection, and $1(5 \%)$ underwent debulking. Eight patients sustained surgical complications (36\%), and two patients $(9 \%)$ died postoperatively. Of the 21 patients, $13(62 \%)$ had either thymic carcinoma or B3 thymoma and $15(71 \%)$ had either Masaoka stage III or IV disease. No patient had a complete pathologic response, but 5 specimens $(24 \%)$ had $<10 \%$ viable tumor.

Conclusions: The present induction chemoradiotherapy protocol, which used specific computed tomography inclusion criteria to successfully select locally advanced thymic tumors, appeared to be tolerable and resulted in a high rate of complete surgical resection. (J Thorac Cardiovasc Surg 2014;147:36-46)

Supplemental material is available online.

\footnotetext{
From the Daniel and Gloria Blumenthal Cancer Center, ${ }^{\mathrm{a}}$ Paramus, NJ; Division of Thoracic Surgery, ${ }^{\mathrm{b}}$ Department of Surgery, The Valley Hospital, Valley Health System, Ridgewood, NJ; The International Thymic Malignancy Interest Group, ${ }^{c}$ Ardsley, NY; Department of Radiation Oncology, ${ }^{\mathrm{d}}$ Princess Margaret Cancer Center, Toronto, Ontario, Canada; Section of Thoracic Surgery, ${ }^{\mathrm{e}}$ Department of Surgery, Methodist Hospital, Houston Tex; Department of Radiation Oncology, ${ }^{f}$ Massachusetts General Hospital, Boston, Mass; Department of Medicine, ${ }^{\mathrm{g}}$ Massachusetts General Hospital, Boston, Mass; Departments of Medicine and Medical Biophysics, ${ }^{\text {h }}$ Princess Margaret Cancer Center, Toronto, Ontario, Canada; Institute of Pathology, ${ }^{\mathrm{i}}$ University Medical Center Mannheim, University of Heidelberg, Mannheim, Germany; Division of Thoracic Surgery, ${ }^{j}$ Department of Surgery, Massachusetts General Hospital, Boston, Mass; and Division of Thoracic Surgery, ${ }^{k}$ Department of Surgery, Toronto General Hospital, Toronto, Ontario, Canada.

This study was funded, in part, by the Valley Hospital Foundation.

Disclosures: Authors have nothing to disclose with regard to commercial support.

Read at the 93rd Annual Meeting of The American Association for Thoracic Surgery, Minneapolis, Minnesota, May 4-8, 2013.

Received for publication April 29, 2013; revisions received Aug 15, 2013; accepted for publication Aug 21, 2013; available ahead of print Oct 17, 2013.

Address for reprints: Robert J. Korst, MD, Division of Thoracic Surgery, Department of Surgery, The Valley Hospital, Valley Health System, 1 Valley Health Plaza, Paramus, NJ 07652 (E-mail: korsro@valleyhealth.com).

$0022-5223 / \$ 36.00$

Copyright (c) 2014 by The American Association for Thoracic Surgery http://dx.doi.org/10.1016/j.jtcvs.2013.08.061
}

Locally advanced thymic epithelial tumors have a significant risk of recurrence after surgical resection, in part, because of the inability to perform a complete resection in approximately $50 \%$ of cases. ${ }^{1}$ Because these tumors have been documented to be chemoresponsive, attempts have been made to increase the resectability of locally advanced thymic tumors using induction chemotherapy. Thus, several retrospective reports and phase II clinical trials with small numbers of patients have been published with results suggesting that the addition of chemotherapy before resection for stage III and IVA thymic tumors might enhance the ability to perform a complete resection with acceptable toxicity compared with historical controls. ${ }^{1-7}$ Despite this, the lack of specific inclusion criteria for these studies has made the comparison of tumor characteristics among the reports impossible.

Given that thymic tumors are also sensitive to radiotherapy, ${ }^{8}$ combined with the importance of complete resection in patient treatment, we designed a prospective, phase II, pilot clinical trial to evaluate the addition of radiotherapy to chemotherapy before resection. Specific radiographic inclusion criteria were also proposed to facilitate the comparison of these trial results with future studies. The purpose of the present trial was to document 


\section{Abbreviations and Acronyms \\ $\mathrm{CPR}=$ complete pathologic response \\ $\mathrm{CT}=$ computed tomography \\ ITMIG = International Thymic Malignancy Interest Group \\ PET = positron emission tomography \\ $\mathrm{SUV}_{\max }=$ maximum standardized uptake value \\ WHO $=$ World Health Organization}

the radiographic and pathologic response to induction chemoradiotherapy, evaluate the toxicity and surgical outcomes, assess the ability of specific radiographic inclusion criteria to predict the tumor stage and histologic type, and document the recurrence patterns and survival.

\section{METHODS \\ Clinical Trial Design}

A single-arm, prospective, pilot clinical trial (NCT00387868) was conducted at 4 institutions and was approved by all 4 institutional review boards. A single site served as the data management center, where the trial was reviewed regularly by the data safety monitoring board. The present trial evaluated an induction therapy regimen of concurrent chemoradiotherapy followed by surgical resection for locally advanced thymic tumors confined to the mediastinum. The study schema is shown in Figure 1.

\section{Patient Population and Inclusion Criteria}

Patients $\geq 18$ years old with a Karnofsky performance status of $\geq 80 \%$ who presented with previously untreated, biopsy-proven thymoma or thymic carcinoma were permitted to participate in the present trial, and all signed the informed consent form. All patients had to have undergone computed tomography (CT) of the chest with intravenous contrast and positron emission tomography (PET) within 60 days before enrollment. The specific CT criteria for accrual included disease confined to the mediastinum and 1 of the following:

Tumors $>8 \mathrm{~cm}$ in the greatest axial diameter

Tumors 5 to $8 \mathrm{~cm}$ in the greatest axial diameter with $\geq 1$ of the following additional criteria:

Irregular or scalloped borders

Heterogeneous appearance

Ectopic calcification

Obvious great vessel and/or adjacent organ invasion or encirclement

Tumors $<5 \mathrm{~cm}$ in the greatest axial diameter with obvious great vessel and/or adjacent organ invasion or encirclement

Patients with pleural or pericardial disease noncontiguous from the primary site (stage IVA) were excluded. When questions arose concerning the eligibility of a given patient, the final decision regarding inclusion was made by the overall study principal investigator (R.J.K.). The number of patients at each institution who met the inclusion criteria but were either not approached to participate or refused to participate was not recorded. Patients were not excluded unless they had not met the inclusion criteria or had met the exclusion criteria. Appendix 1 provides a comprehensive list of the inclusion and exclusion criteria.

\section{Treatment Regimen}

The induction therapy consisted of cisplatin, etoposide, and concurrent radiotherapy $\leq 4500 \mathrm{cGy}$. After chemoradiotherapy, the patients underwent restaging with contrast-enhanced CT and PET. All patients without distant progression were eligible for an attempt at surgical resection. The operative approach was left to the discretion of the individual surgeon; however, consistent attempts were made to perform an en bloc resection whenever possible and to document close margins using frozen section analysis. Resection occurred within a 4 to 8 -week interval after the completion of radiotherapy. Debulking was allowed for tumors deemed to be grossly unresectable at surgery. Patients underwent postoperative therapy in accordance with the study schema (Figure 1). The conduct of the induction regimen and postoperative treatment plan has been detailed in Appendix 2.

\section{Study Endpoints and Response Assessment}

The primary endpoint was the rate of the complete pathologic response (CPR) to the preoperative chemoradiotherapy regimen. Thus, the pathologic evaluation of the surgical specimens, which was performed by the individual institutional pathologists, included not only the assignment of the World Health Organization (WHO) histologic type but also an estimation of the percentage of viable tumor in the specimen. At the time of trial design, no standard protocol existed for the assessment of viability in thymic tumors; therefore, this was performed according to the individual institutional protocols and the judgment of the reading pathologist. Patients with $\leq 10 \%$ of their tumor mass composed of viable tumor were designated as having a "near CPR." A post hoc examination of all available tissue blocks and slides from the resected specimens was performed in a blinded fashion by an independent pathology representative (A.M.) of the International Thymic Malignancy Interest Group (ITMIG) in an attempt to corroborate the WHO histologic type. However, the institutional pathology assessment was used for all analyses in the present clinical trial, as per the protocol. The secondary endpoints of the study were

The ability of the predetermined CT criteria and maximum standardized uptake value $\left(\mathrm{SUV}_{\max }\right)$ to predict the Masaoka stage ${ }^{10}$ and WHO histologic type

The rate of complete resection

Toxicity and surgical complications

The radiographic response to induction chemoradiotherapy using CT and PET

Recurrence rates, failure patterns, and survival

A complete resection (R0) was defined as negative gross and microscopic margins. Incomplete resections were divided into microscopically positive margins (R1) and grossly positive margins (R2). Debulking was considered an R2 resection.

The radiographic response was measured using CT and PET. The tumor size was assigned according to the maximal diameter of the tumor as measured in the axial plane on the CT scan. The post-treatment and pretreatment sizes were compared, and the responses were classified according to the Response Evaluation Criteria in Solid Tumors criteria. ${ }^{11}$ In addition, the magnitude of the response was calculated for each patient using the formula: [(post-treatment size) - (pretreatment size)/pretreatment size $] \times 100 \%$. The magnitude of the response to induction therapy was also measured using PET using the formula: [(post-treatment $\mathrm{SUV}_{\max }$ ) - (pretreatment $\mathrm{SUV}_{\max }$ )/pretreatment $\left.\mathrm{SUV}_{\max }\right] \times 100 \%$.

The toxicity of the chemoradiotherapy regimen was defined using the Common Terminology Criteria for Adverse Events, version 4. Surgical complications were defined separately, but not graded. After the treatment portion of the protocol, the patients were followed up every 6 months with an interim medical history, a physical examination, PET, and CT. 


\section{Biopsy-proven thymoma or thymic carcinoma}

-Specific radiographic criteria

- No evidence of distant metastases

-CT and PET scans

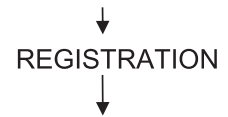

CISPLATIN $\left(50 \mathrm{mg} / \mathrm{m}^{2}\right)$ Days $1,8,29,36$

ETOPOSIDE $\left(50 \mathrm{mg} / \mathrm{m}^{2}\right)$ Days $1-5,29-33$

CONCURRENT RADIOTHERAPY (IMRT or 3D conformal) Weeks 1-5 (M-F)

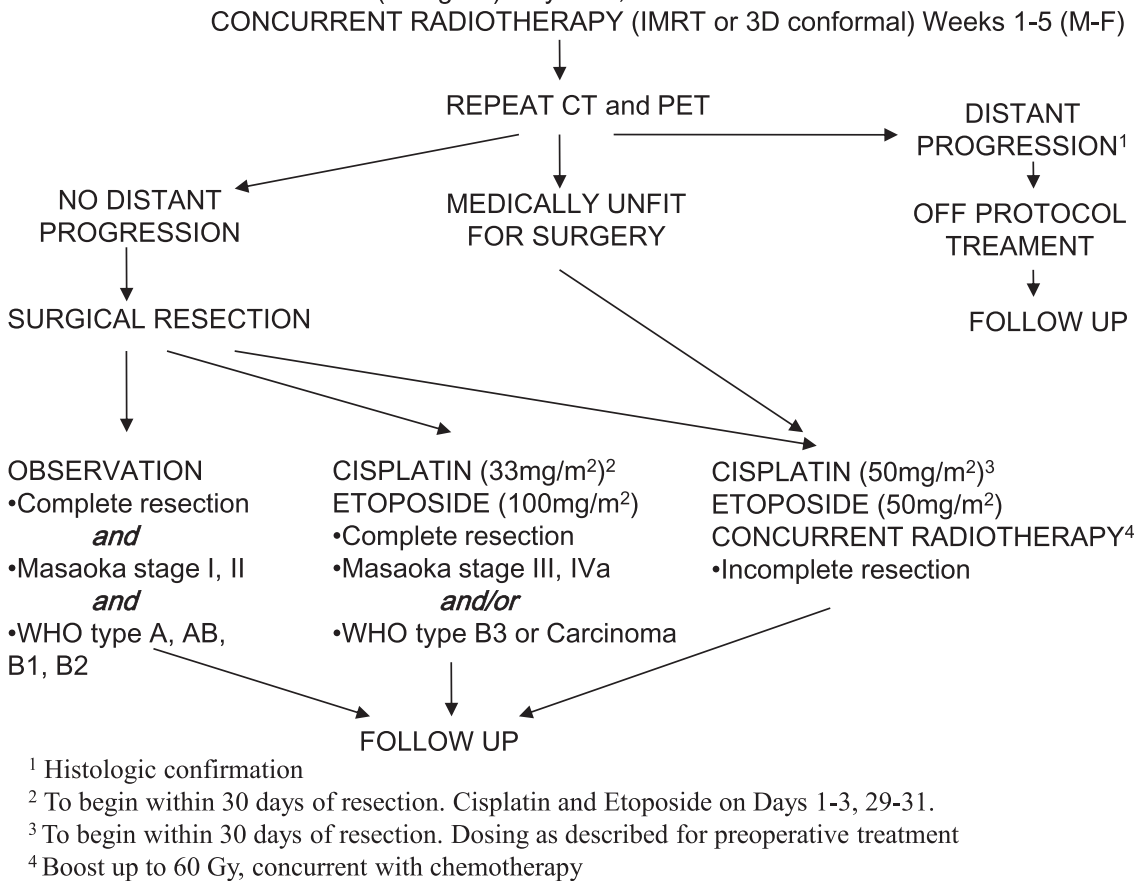

FIGURE 1. Study schema. $C T$, Computed tomography; $P E T$, positron emission tomography; IMRT, intensity-modulated radiotherapy; $3 D$, 3-dimensional; $M$, Monday; $F$, Friday; $W H O$, World Health Organization.

\section{Statistical Analysis}

Because of the small study size, all comparisons between medians were performed using nonparametric tests. The Kruskal-Wallis test was used to assess for significance among the medians of 3 groups of data, and the Wilcoxon-Mann-Whitney test was used to assess for significance between the medians of 2 groups. All comparisons were 2-tailed. The follow-up data were analyzed using the Kaplan-Meier method, and the overall and disease-free survival from the date of accrual were plotted. IBM-SPSS Statistics software, version 19 (SPSS, Armonk, NY) was used for the statistical analysis.

\section{RESULTS}

\section{Initial Radiographic Characteristics}

A total of 22 patients were accrued at 4 institutions during a 5-year period (July 2007 through June 2012). The median tumor size on the CT scan was $6.8 \mathrm{~cm}$ (range, 5-14). A summary of the CT inclusion criteria encountered is presented in Table 1, and the radiographic features of each individual tumor from the 21 patients are listed in Table E1, along with the associated stage and WHO histologic type. All tumors possessed abnormal uptake on the initial PET scan, with a median initial $\mathrm{SUV}_{\max }$ of
5.1 (range, 1.5-15). Two patients did not have the $\mathrm{SUV}_{\max }$ reported, but their tumors were noted to be hypermetabolic. Figure 2 demonstrates the relationship between the initial $\mathrm{SUV}_{\max }$ and the final WHO histologic type. Patients with thymic carcinoma $(n=5)$ had a higher initial $\mathrm{SUV}_{\max }$ than did those with type B3 thymoma $(n=6)$ or the group with $\mathrm{A}, \mathrm{AB}, \mathrm{B} 1, \mathrm{~B} 2$, and metaplastic thymoma $(\mathrm{n}=8$; $P=.003)$. No significant differences in the initial $\mathrm{SUV}_{\max }$ were appreciated between the tumors postoperatively determined to be stage I and II ( $\mathrm{n}=6$; median $\left.\mathrm{SUV}_{\max }, 4.1\right)$ versus stage III and IV $\left(\mathrm{n}=13\right.$; median $\mathrm{SUV}_{\max }, 5.3$; $P=.41)$.

\section{Conduct of Protocol and Complete Resection Rate}

Of the initial 22 patients, 1 patient withdrew from the study before beginning the induction therapy protocol. Of the 21 patients, 17 were men $(80 \%)$, and the median age was 51 years (range, 18-78). Five patients had myasthenia gravis $(24 \%)$, and a single patient had pure red blood cell aplasia $(5 \%)$. Figure 3 demonstrates the Consolidated 
TABLE 1. Radiographic inclusion criteria, thoracic structures resected, WHO histologic type, and Masaoka stage of 21 patients undergoing attempted resection

\begin{tabular}{lc}
\hline \multicolumn{1}{c}{ Variable } & Patients (n) \\
\hline Radiographic inclusion criteria & \\
Tumor $>8 \mathrm{~cm}$ & $6(29)$ \\
Tumor 5-8 cm & \\
$\quad 1$ criterion & $5(24)$ \\
2 criteria & $7(33)$ \\
$\quad 3$ criteria & $3(14)$ \\
Tumor $<5$ cm & 0 \\
Resected thoracic structures & \\
Pericardium & $8(38)$ \\
Lung* & $8(38)$ \\
Phrenic nerve & $7(33)$ \\
Superior vena cava & $2(10)$ \\
Diaphragm & $2(10)$ \\
Vagus nerve & $1(5)$ \\
Innominate artery & $1(5)$ \\
Sternum & $1(5)$ \\
Ribs & $1(5)$ \\
None & $5(24)$ \\
WHO histologic type & \\
Metaplastic & $1(5)$ \\
A & $2(10)$ \\
AB & $1(5)$ \\
B1 & $1(5)$ \\
B2 & $3(14)$ \\
B3 & $6(29)$ \\
Postoperative Masaoka stage & $7(33)$ \\
II & $2(10)$ \\
III & $4(19)$ \\
IVA & $12(57)$ \\
IVB & $1(5)$ \\
\hline Detana & $2(10)$ \\
\hline
\end{tabular}

Data in parentheses are percentages. WHO, World Health Organization. *Seven wedge resections, one left pneumonectomy

Standards of Reporting Trials diagram detailing the flow of patients through the protocol. Of the 21 patients who completed preoperative radiotherapy, 3-dimensional conformal techniques were used in 6 patients $(29 \%)$ and intensity-modulated radiotherapy in 15 . The total preoperative radiotherapy dose ranged from 4000 to $4500 \mathrm{cGy}$, with 17 patients $(81 \%)$ receiving the maximum of $4500 \mathrm{cGy}$. Surgical resection was attempted through a median sternotomy in 19 ( 1 reoperative), a partial sternotomy with anterior thoracotomy (hemiclamshell) in 1, and bilateral anterior thoracotomy with a transverse sternotomy (clamshell) in 1 patient. The additional thoracic structures resected in these 21 patients are listed in Table 1. On an intention to treat basis, 17 patients $(77 \%)$ underwent an $\mathrm{R} 0$ resection, 3 $(14 \%)$ an $\mathrm{R} 1$ resection, and 1 underwent debulking (5\%). Of the 3 patients who underwent an R1 resection, 1 had B3 thymoma and gross phrenic nerve invasion. Although

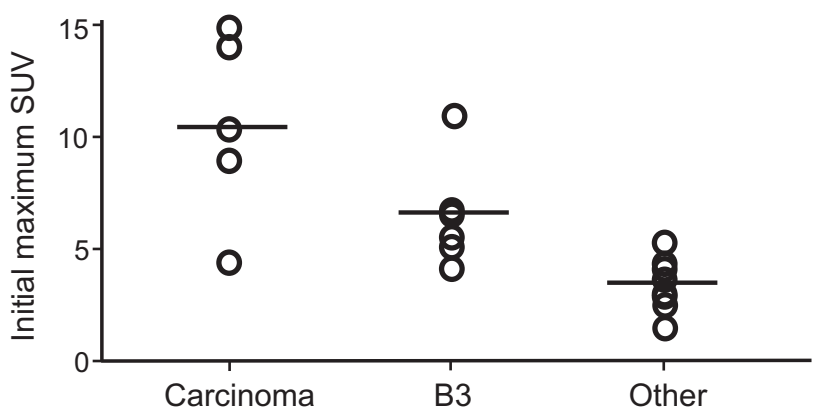

FIGURE 2. Pretreatment maximum standard uptake value (SUV) from 21 patients with thymic epithelial tumors and its relationship to the World Health Organization histologic type. Each data point represents an individual patient, and the horizontal bars represent the median values. Two patients with carcinoma did not have a maximum standard uptake value reported. $P=.14$, carcinoma versus $\mathrm{B} 3 ; P=.005$, carcinoma versus other; $P=.008, \mathrm{~B} 3$ versus other.

an $\mathrm{R} 0$ resection could have been achieved by resecting the phrenic nerve, an intraoperative decision was made not to resect this nerve because of the patient's history of generalized myasthenia gravis, leaving a microscopically positive margin. The single debulking was performed in 1 patient with clear encirclement of the ascending aorta with tumor.

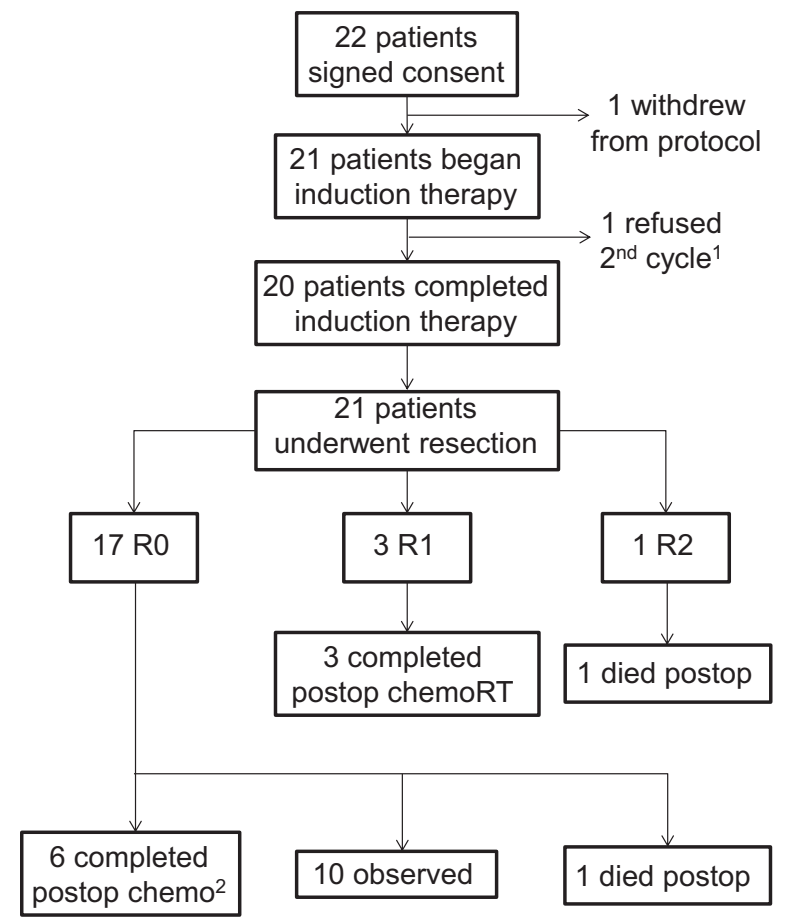

1 due to myasthenia exacerbation

2 four additional patients did not complete postop chemotherapy due to patient refusal $(n=3)$ and postop neutropenia $(n=1)$

FIGURE 3. Consolidated Standards of Reporting Trials diagram showing the flow of patients through the protocol. Postop, Postoperative; chemoRT, chemoradiotherapy; chemo, chemotherapy. 
TABLE 2. Toxicity of induction chemoradiotherapy and surgical complications

\begin{tabular}{ll}
\hline \multicolumn{1}{c}{ Variable } & Patients (n) \\
\hline Induction therapy toxicity* $\dagger$ & \\
Grade 3 & \\
$\quad$ Tinnitus & $1(5,0-13)$ \\
Allergic reaction & $1(5,0-13)$ \\
Dehydration & $1(5,0-13)$ \\
Myasthenia gravis $\ddagger$ & $1(5,0-13)$ \\
Esophagitis & $1(5,0-13)$ \\
Nausea and vomiting & $1(5,0-13)$ \\
Febrile neutropenia & $2(9,0-21)$ \\
Grade 4 & \\
Thrombocytopenia & $1(5,0-13)$ \\
Cardiac arrest & $1(5,0-13)$ \\
Surgical complications $\dagger$ & \\
Pneumonia & $1(5,0-13)$ \\
Pulmonary infiltrate & $1(5,0-13)$ \\
Hemothorax & $1(5,0-13)$ \\
Mucous plugging & $1(5,0-13)$ \\
Aspiration & $1(5,0-13)$ \\
Pleural effusion & $1(5,0-13)$ \\
Intraoperative cardiac arrest & $1(5,0-13)$ \\
Multiple organ failure & $1(5,0-13)$ \\
Neutropenia & $1(5,0-13)$ \\
Dressler syndrome & $2(9,0-21)$ \\
Death & $2(9,0-21)$ \\
Atrial fibrillation & $3(14,0-28)$ \\
\hline
\end{tabular}

Data presented as number of patients, with the percentages and $95 \%$ confidence intervals in parentheses. *According to Common Terminology Criteria for Adverse Events, version 4. †Percentages calculated using intention to treat. $\ddagger$ Exacerbation during induction therapy.

\section{Toxicity and Surgical Complications}

Toxicity from the induction therapy regimen (grades 3 and 4) occurred in 9 patients $(41 \%$ according to the intention to treat; Table 2). Grade 4 thrombocytopenia developed in the single patient who had initially presented with pure red blood cell aplasia in addition to thymoma. The grade 4 cardiac arrest (from which the patient was successfully resuscitated) was experienced by 1 patient in the postanesthesia care unit after implantable port placement for chemotherapy.

Surgical complications occurred in 8 patients $(36 \%$; Table 2). One of the postoperative deaths occurred in a patient with a $7.3-\mathrm{cm}$ thymic carcinoma invading the left pulmonary hilum for which pneumonectomy and resection of both the phrenic and the vagus nerves were required to achieve an $\mathrm{R} 0$ resection. The patient aspirated in the postoperative period and died of respiratory failure. The second death occurred in the patient with a $10.3-\mathrm{cm}$ B3 thymoma who had undergone debulking because of encirclement of the ascending aorta. This patient sustained an intraoperative cardiac arrest of unclear etiology with subsequent postoperative multiple organ system failure.

\section{Radiographic and Pathologic Response to Induction Chemoradiotherapy}

Although 20 of 21 patients experienced some degree of radiographic tumor shrinkage from the induction therapy regimen as determined by CT (Table E1), only 10 patients met the criterion for a partial radiographic response according to the Response Evaluation Criteria in Solid Tumors. An additional 11 patients had stable disease, no patient progressed radiographically, and no patient achieved a complete radiographic response. A trend was seen toward a greater magnitude of radiographic response as measured by the tumor size (Figure 4, A) in the 7 patients with thymic carcinoma compared with the 8 patients with $\mathrm{A}, \mathrm{AB}, \mathrm{B} 1, \mathrm{~B} 2$, and metaplastic thymoma $(-28 \%$ vs $-15 \% ; P=.09)$.

The $\mathrm{SUV}_{\text {max }}$ decreased in 18 patients and increased in 1 patient after induction chemoradiotherapy (Table E1).

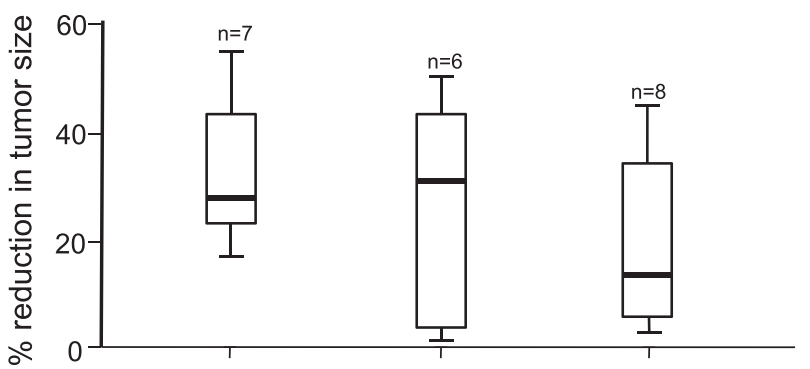

A

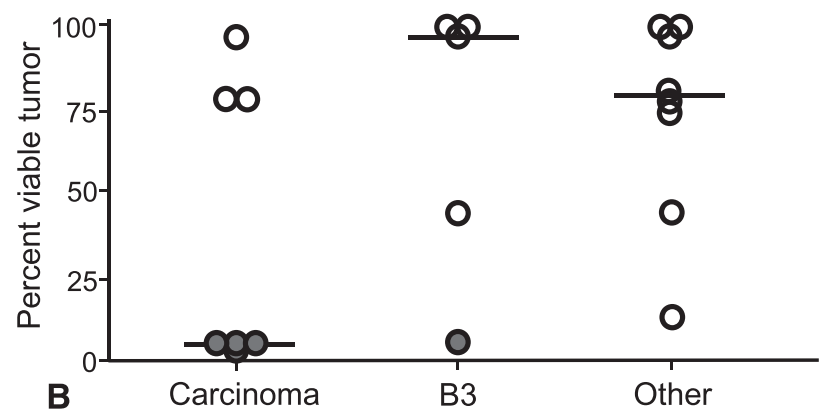

FIGURE 4. The relationship between the World Health Organization histologic type and response to induction therapy. "Other" refers to type $\mathrm{A}, \mathrm{AB}, \mathrm{B} 1, \mathrm{~B} 2$, and metaplastic thymoma. A, Box and whisker plot of the magnitude of response to induction chemoradiotherapy measured by the tumor size on the computed tomography scan. The upper and lower ends of each box represent the upper and lower quartiles, the thick horizontal bar represents the median, and the extreme horizontal bars represent the maximum and minimum values. $P=.09$, carcinoma versus other; $P=.67$, carcinoma versus B3; $P=.56$, B3 versus other. $\mathrm{B}$, Pathologic response to induction chemoradiotherapy reported as the percentage of viable tumor in the surgical specimens as estimated by the institutional pathologists. Each data point represents an individual patient and the horizontal bars represent the median values. The solid points represent patients with a near complete pathologic response. One patient with a B3 thymoma did not have an estimate of the percentage of viable tumor reported. $P=.14$, carcinoma versus B3; $P=.07$, carcinoma versus other; $P=.94, \mathrm{~B} 3$ versus other. 

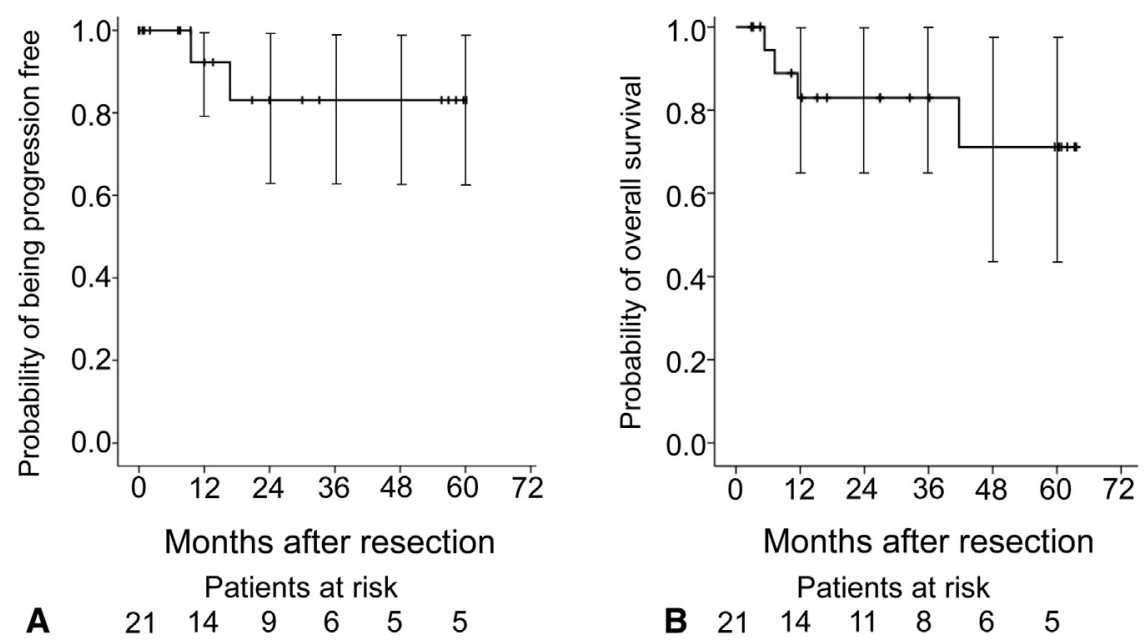

FIGURE 5. Freedom from progression and actuarial overall survival after multimodality treatment of locally advanced thymoma and thymic carcinoma. The vertical bars indicate $95 \%$ confidence intervals. A, Freedom from progression. B, Overall survival.

Two additional patients did not have a $\mathrm{SUV}_{\max }$ reported in their PET scan readings. The median magnitude of change in the $\mathrm{SUV}_{\max }$ after induction therapy was $-44.5 \%$, with 5 patients $(24 \%)$ having complete normalization of their PET scans after induction therapy ( 2 with carcinoma, 2 with type B2, and 1 with type B1). Despite this normalization, only 2 of these (both with carcinoma) had a near CPR. No significant differences were noted in the median magnitude of the response as measured using the $\mathrm{SUV}_{\max }$ according to the WHO histologic type (carcinoma, -39\%; B3 thymoma, $-45 \%$; $, \mathrm{AB}, \mathrm{B} 1, \mathrm{~B} 2$, and metaplastic thymoma, $-43.5 \% ; P=.91)$.

The postoperative Masaoka stage and WHO histologic type (from the institutional pathologists) of the 21 patients who underwent attempted resection are listed in Table 1. All patients had viable tumor in their surgical specimen (Table E1). The relationship between the WHO histologic type and pathologic response to the induction regimen is displayed in Figure 4, $B$. Despite the lack of any CPR to the induction chemoradiotherapy, 5 patients $(24 \%)$ were deemed to have a near CPR.

Paraffin-embedded tissue blocks or slides were made available for 16 of the 21 resected specimens for the blinded ITMIG pathologist to perform a post hoc review; however, only 15 of these had enough material to satisfactorily establish the WHO histologic type. In 9 of 15 cases, the ITMIG pathology assessment was concordant with the institutional pathology report, with 6 discordant. In 4 of the discordant cases, the difference in the interpretation was considered "minor" (eg, B2 vs $\mathrm{B} 1$; $\mathrm{A}$ vs $\mathrm{AB}$ ). In the other 2 discordant cases (A vs B2; A vs B3), the ITMIG pathologist performed additional immunohistochemistry testing (CD20), which led to the discordance.

\section{Recurrence and Survival}

With a median follow-up of 27 months (range, 0-64) for the entire treated cohort, no patient who underwent a complete resection had experienced recurrence. Thus, the freedom from recurrence rate was $100 \%$ in the completely resected patients. Of the 19 patients who survived into the follow-up period, 15 were alive without recurrence, 1 was alive with persistent disease (pleura, liver), 1 had died of persistent and/or progressive tumor (lung, mediastinum, pulmonary hilum), 1 had died of pneumonia without recurrence, and 1 was lost to follow-up. Both patients with persistent and/or progressive disease had undergone an $\mathrm{R} 1$ resection and had received chemotherapy once they had developed progression. At 60 months after the date of resection, the freedom from progression and overall actuarial survival of the entire 22-patient cohort was $83 \%$ and $71 \%$, respectively (Figure 5).

\section{DISCUSSION \\ Utility of CT and PET in Predicting Masaoka Stage and WHO Histologic Type}

The Masaoka staging system relies on information obtained at the resection and pathologic examination, making the preoperative identification of Masaoka stage III thymic tumors inconsistent at best. Thus, previously published induction therapy trials have been difficult to compare with each other, because no specific radiographic inclusion criteria were used. Also, the tumors have generally been described using highly subjective terms (eg, "unresectable"). ${ }^{2,3}$

In the present trial, specific $\mathrm{CT}$ radiographic criteria were developed in an attempt to preoperatively identify patients with tumors at greater risk of recurrence ("high-risk" disease) and hopefully standardize the inclusion of patients 
to locally advanced thymic tumor clinical trials. These criteria were determined from previously published CT features of invasive thymoma, ${ }^{12}$ some of which are subsequently being evaluated by the ITMIG. ${ }^{13}$ Of the 21 patients, $71 \%$ were confirmed to have locally advanced tumors (Masaoka stage III and IV) when these criteria were applied, a percentage that was likely greater given that patients all had undergone chemoradiotherapy before staging (Table 1). Similarly, these criteria also seemed to select patients with high-risk tumors according to the WHO histologic type, with $62 \%$ having either thymic carcinoma or B3 thymoma. These findings suggest that specific CT criteria can be used to identify patients with high-risk thymic tumors.

The ability of PET to identify patients with high-risk tumors was also evaluated in the present trial. Although the use of $\mathrm{SUV}_{\max }$ as a quantitative measure for risk stratification of malignant tumors has been associated with inherent subtle variations in scan technique and biologic variables, which can affect its value, ${ }^{14}$ the presented data showed a striking relationship between the $\mathrm{SUV}_{\text {max }}$ and WHO histologic type, with the highest risk tumors possessing the greater $\mathrm{SUV}_{\max }$ values, despite the small sample sizes (Figure 2). This observation has been previously reported in some retrospective studies. ${ }^{15-17}$ From these data, the use of PET to stratify preoperative risk in patients with thymic tumors is worthy of additional evaluation.

\section{Addition of Radiotherapy to Induction Chemotherapy}

The rationale for the addition of radiotherapy to induction chemotherapy in the present trial was to attempt to enhance the rate of complete resection and the pathologic response compared with induction chemotherapy alone. ${ }^{18}$ The observation that only 6 patients did not meet the criteria for a radiographic response or a near $\mathrm{CPR}$, yet still underwent an R0 resection, suggests that most patients might have benefitted from the induction regimen.

The 2 largest prospective clinical trials published to date using induction chemotherapy alone for thymoma reported a complete resection rate of $43 \%$ ( 9 of 21 ) and $73 \%$ (16 of 22) when analyzed according to the intention to treat. ${ }^{2,3}$ The results from the present study compare favorably with the former 2 studies at 77\% (17 of 22); however, these 3 trials differed significantly in multiple factors, including the radiographic and pathologic inclusion criteria, Masaoka stage, chemotherapeutic agents, and assessment of the radiographic response. In the Japanese Clinical Oncology Group 9606 trial, ${ }^{3}$ the patients had "unresectable" tumor localized to the mediastinum, but patients with thymic carcinoma were excluded. Similarly, Kim and colleagues ${ }^{2}$ included patients deemed "unresectable" but also included patients with stage IVA and excluded those with thymic carcinoma.

The toxicity of the induction chemoradiotherapy regimen was generally acceptable, with all but 1 patient completing the regimen. This was similar to the induction chemotherapy study reported by Kim and colleagues, ${ }^{2}$ in which 20 of 22 patients completed the induction regimen. However, it was significantly different from the Japanese Clinical Oncology Group 9606 trial in which only 57\% of patients completed the induction chemotherapy. ${ }^{3}$ Treatment-related mortality in the present study consisted exclusively of 2 postoperative deaths that appeared to be related to the surgical procedure itself and not the preoperative treatment regimen. In contrast, the single treatment-related death report by Kim and colleagues ${ }^{2}$ occurred during the adjuvant therapy portion of the regimen. The Japanese Clinical Oncology Group 9606 trial $^{3}$ reported no treatment-related mortality.

An unexpected finding was the lack of any CPR to induction chemoradiotherapy in the present trial, despite $24 \%$ of the patients having a near CPR. The 2 previously discussed trials reported a CPR rate of $10 \%$ and $14 \%$ using chemotherapy alone., ${ }^{2,3}$ Intuitively, it would be expected that the addition of radiotherapy would increase the number of CPRs, but this did not occur. Explanations for this finding include differences in the chemotherapy regimens (although all were cisplatin-based), the pathologic assessment, and, possibly, differences in the histologic type, although this remains to be determined. The present study revealed a trend toward a greater magnitude of response (as measured by CT) in the patients with thymic carcinoma compared with the group with $\mathrm{A}, \mathrm{AB}, \mathrm{B} 1, \mathrm{~B} 2$, and metaplastic thymomas (Figure 4, $A)$. This is even more intriguing when one considers that 4 of the 5 patients with a near CPR had thymic carcinoma. These findings imply that patients with high-risk WHO histologic types might respond the best to this induction chemoradiotherapy protocol. This observation is worthy of additional study and clearly emphasizes the need to appropriately select future high-risk patients for induction therapy.

\section{Study Strengths and Limitations}

The clear strength of the present study is that it represents a formal, prospective clinical trial for a rare disease with rigorous data collection and quality. An additional strength was the objective CT criteria used for patient selection. By avoiding subjective terms such as "unresectable," these inclusion criteria can be applied to future locally advanced thymoma trials and the outcomes more readily compared.

A major limitation of the present study was the small sample size, not surprising, given the rarity of these tumors. This small sample size limited some of the conclusions that can be drawn from the data such as a strict definition of a 
"cutoff" value for the SUV $_{\max }$ for high-risk tumors. Another limitation was in the ability of the imaging criteria to predict the Masaoka stage. Because the patients had received induction therapy, their stage could have changed by the time they underwent resection. Despite this, it seems more likely than not that these patients' disease did not progress during the induction protocol, because no tumors had enlarged, and the vast majority had regressed in size. Therefore, it follows that the patients' disease stages were at least as advanced at presentation compared with at surgery. Finally, the pathologic examinations were limited by the use of individual institutional pathology examinations. Ultimately, the best study design would be to have a single pathologist review all the specimens and use an established protocol for estimating the amount of viable tumor in each specimen. However, no such protocol for thymic tumors existed when the present trial was designed.

\section{CONCLUSIONS}

The present study has demonstrated that induction chemoradiotherapy is a viable option for patients with locally advanced thymic tumors and that its use results in a high rate of complete surgical resection. In addition, specific CT findings (ie, large tumor size, irregular borders, heterogeneous appearance, ectopic calcification, obvious great vessel or adjacent organ invasion and/or encirclement) and PET criteria (high $\mathrm{SUV}_{\max }$ ) appear to be able to select patients who ultimately have locally advanced Masaoka stages and high-risk histologic types (B3 and carcinoma).

\section{References}

1. Detterbeck FC, Parsons AM. Thymic tumors. Ann Thorac Surg. 2004:77:1860-9.

2. Kim ES, Putnam JB, Komaki R, Walsh GL, Ro JY, Shin HJ, et al. Phase II study of a multidisciplinary approach with induction chemotherapy, followed by surgical resection, radiation therapy, and consolidation chemotherapy for unresectable malignant thymomas: final report. Lung Cancer. 2004;44:369-79.

3. Kunitoh H, Tamura T, Shibata T, Takeda K, Katakami N, Nakagawa K, et al. A phase II trial of dose-dense chemotherapy, followed by surgical resection and/or thoracic radiotherapy, in locally advanced thymoma: report of a Japan Clinical Oncology Group trial (JCOG 9606). Br J Cancer. 2010;103:6-11.

4. Lucchi M, Ambrogi MC, Duranti L, Basalo F, Fontanini G, Angeletti CA, et al. Advanced stage thymomas and thymic carcinomas: results of multimodality treatments. Ann Thorac Surg. 2005;79:1840-4.

5. Venuta F, Rendina EA, Longo F, De Giacomo T, Anile M, Mercadante E, et al. Long-term outcome after multimodality treatment for stage III thymic tumors. Ann Thorac Surg. 2003;76:1866-72.

6. Rea F, Sartori F, Loy M, Calabro F, Fornasiero A, Daniele O, et al. Chemotherapy and operation for invasive thymoma. J Thorac Cardiovasc Surg. 1993; 106:543-9.

7. Macchiarini P, Chella A, Ducci F, Rossi B, Testi C, Bevilacqua G, et al. Neoadjuvant chemotherapy, surgery, and postoperative radiation for invasive thymoma. Cancer. 1991;68:706-13.

8. Onuki T, Ishikawa S, Yamamoto T, Ito H, Sakai M, Onizuka M, et al. Pathologic radioresponse of preoperatively irradiated invasive thymomas. J Thorac Oncol. 2008:3:270-6.

9. Rosai J, Sobin L. Histologic typing of tumors of the thymus. In: World Health Organization, ed. International Histological Classification of Tumors. 2nd ed. New York: Springer; 1999:9-14.

10. Masaoka A, Monden Y, Nakahara K, Tanioka T. Follow-up study of thymomas with special reference to their clinical stages. Cancer. 1981;48:2485-92.
11. Eisenhauer EA, Therasse P, Bogaerts J, Schwartz LH, Sargent D, Ford R, et al New response evaluation criteria in solid tumors: revised RECIST guideline (version 1.1). Eur J Cancer. 2009; 45:228-47.

12. Tomiyama N, Muller NL, Ellis SJ, Cleverley JR, Okumura M, Miyoshi S, et al. Invasive and noninvasive thymoma: distinctive CT features. J Comput Assist Tomogr. 2001;25:388-93.

13. Marom EM, Rosado-de-Christenson ML, Bruzzi JF, Hara M, Sonnett JR, Ketai L. Standard report terms for chest computed tomography reports of anterior mediastinal masses suspicious for thymoma. J Thorac Oncol. 2011;6:S1717-23.

14. Adams MC, Turkington TG, Wilson JM, Wong TZ. A systematic review of the factors affecting accuracy of SUV measurements. AJR Am J Roentgenol. 2010; 195:310-20.

15. Fukumoto K, Taniguchi T, Ishikawa Y, Kawaguchi K, Fukui T, Matsuo K, et al. The utility of $[18 \mathrm{~F}]$-fluorodeoxyglucose positron emission tomographycomputed tomography in thymic epithelial tumors. Eur J Cardiothorac Surg. 2012;42:e152-6

16. Terzi A, Bertolaccini L, Rizzardi G, Luzzi L, Bianchi A, Campione A, et al Usefulness of 18-F FDG PET/CT in the pre-treatment evaluation of thymic epithelial neoplasms. Lung Cancer. 2011;74:239-43.

17. Benveniste MF, Moran CA, Mawlawi O, Fox PS, Swisher SG, Munden RF, et al FDG PET-CT aids in the preoperative assessment of patients with newly diagnosed thymic epithelial malignancies. J Thorac Oncol. 2013;8:502-10.

18. Wright CD, Choi NC, Wain JC, Mathisen DJ, Lynch TJ, Fidias P. Induction chemoradiotherapy followed by resection for locally advanced Masaoka stage III and IVA thymic tumors. Ann Thorac Surg. 2008;85:385-9.

\section{APPENDIX 1. INCLUSION CRITERIA}

Age $\geq 18$ years

Male or female

Karnofsky performance status $>80 \%$

Biopsy-proven thymoma, including carcinoma; biopsy could be obtained by multiple approaches, including fine needle aspiration for cytology, transcutaneous core needle biopsy, or open, thoracoscopic, or mediastinoscopic biopsy, with the latter 2 techniques preferred

Biopsy slides must be reviewed by pathologists at participating institutions and assigned a WHO classification wherever possible based on the biopsy material

Patients must have a CT scan of the chest with intravenous contrast within 60 days of enrollment

Tumors $>8 \mathrm{~cm}$ in the greatest diameter on the CT scan

For tumors $5-8 \mathrm{~cm}$ in the greatest diameter on the CT scan, $\geq 1$ of the following radiographic criteria must also be present on the intravenous contrastenhanced CT scan:

Multifocal calcification

Heterogeneous appearance

Irregular or scalloped borders

Obvious great vessel invasion or encirclement

For tumors $<5 \mathrm{~cm}$ in the greatest diameter on the CT scan, obvious great vessel invasion and/or encirclement must be present on the CT scan

Patients with anterior mediastinal carcinoma unable to be further differentiated and who have no evidence 
of another primary tumor site or metastatic spread beyond the mediastinum

Pulmonary function acceptable for surgery according to institutional criteria

Laboratory criteria (within 30 days of enrollment):

Absolute neutrophil count $\geq 1500 / \mu \mathrm{L}$

Platelet count $\geq 100,000$

Total bilirubin and aspartate aminotransferase/alanine aminotransferase $\leq 1.5$ times the institutional upper limit of normal

Creatinine clearance $\geq 50 \mathrm{~mL} / \mathrm{min}$ (calculated using institutional standard)

\section{Exclusion Criteria}

Patients thought to be unable to medically tolerate surgical resection as assessed at the initial presentation

Hypersensitivity to cisplatin and/or etoposide

Patients with radiographic evidence of stage IVA thymoma (pleural or pericardial disease noncontiguous with the primary tumor site)

Pretreatment biopsy showing WHO type A thymoma when reviewed by institutional pathologists, unless obvious great vessel invasion and/or encirclement is present on the CT scan; it is understood that a WHO subtype might not be able to be assigned on the basis of the biopsy specimen in all cases

Previous radiotherapy to the chest that would preclude the administration of radiation using 3-dimensional conformal or intensity-modulated radiotherapy planning

Significant medical or psychiatric illness that would interfere with patient compliance

Patients whose 3-dimensional radiotherapy plan cannot meet the dose-volume constraint

Patients receiving other investigational drugs

Pregnant women or women of childbearing potential (women are not considered of childbearing potential if they are $\geq 2$ years postmenopausal and/or surgically sterile) who refuse to use effective contraception; will be determined on the judgment of the institutional principal investigators or designated associates

Failure to meet inclusion criteria

\section{APPENDIX 2. INDUCTION REGIMEN}

Cisplatin was administered by continuous intravenous infusion for 1 hour on days $1,8,29$, and 36 at a dose of $50 \mathrm{mg} / \mathrm{m}^{2}$. The patients were hydrated both before and after cisplatin dosing with mannitol and saline. The use of colony-stimulating factors was prohibited. Etoposide was administered by continuous intravenous infusion for 1 hour on days 1 to 5 and 29 to 33 at a dose of $50 \mathrm{mg} / \mathrm{m}^{2}$. Dosage modifications, including reductions and delays, were incorporated into the protocol for hematologic toxicity, if the creatinine clearance decreased to $<50 \mathrm{~mL} /$ min, and/or grade 3 or 4 nonhematologic toxicity.

Preoperative radiotherapy began within 24 hours of chemotherapy initiation using either a 3-dimensional conformal-or intensity-modulated radiotherapy-based plan. The radiation dose was 4000 to $4500 \mathrm{cGy}$ at 180 to $200 \mathrm{cGy} /$ fraction (weeks 1-5; Monday through Friday). The treatment target included the entire thymus and the gross tumor, with a 2 to $2.5-\mathrm{cm}$ margin. To minimize toxicity, dose-volume constraints were incorporated into the radiation plan for the lungs, esophagus, heart, and spinal cord. Radiotherapy interruptions were permitted for febrile neutropenia, grade 4 mucositis or esophagitis, and grade 3-4 pulmonary toxicity.

\section{Postoperative Treatment}

Patients undergoing adjuvant chemotherapy alone received cisplatin by continuous intravenous infusion for 1 hour on days 1 to 3 and 29 to 31 at a daily dose of $33 \mathrm{mg} / \mathrm{m}^{2}$. Patients were hydrated both before and after cisplatin dosing with mannitol and saline. Etoposide was administered by continuous intravenous infusion for 1 hour on days 1 to 3 and 29 to 31 at a dose of $100 \mathrm{mg} / \mathrm{m}^{2}$. Patients undergoing adjuvant chemoradiotherapy (patients with incomplete resection) received the same treatment protocol used in the preoperative setting, except that only an additional 2000 to $2500 \mathrm{cGy}$ of radiation (180 to $200 \mathrm{cGy} /$ fraction; ending during the third treatment week) was administered to the sites of residual disease. In these cases, the chemotherapy dose for the second postoperative cycle was increased to the level administered in the absence of radiotherapy (cisplatin $33 \mathrm{mg} / \mathrm{m}^{2}$ and etoposide $100 \mathrm{mg} / \mathrm{m}^{2}$ on days $29-31$ ).

\section{Discussion}

Dr Frank C. Detterbeck (New Haven, Conn). Bob, nice presentation. I want to thank you for pulling this off. This was really Bob's trial that he initiated, and I think to pull off a multicenter trial with multiple institutions and a rare disease such as thymoma is really an accomplishment, and I think that is great. There really are not all that many prospective series out there. I think that is 1 of the strengths of this study-it is a multicenter trial, and it is prospective. I think another major strength is that the patients are really quite well characterized by the radiographic findings. I think all too often we are limited by reading reports. One walks away from the report, saying, "well, that is interesting, but I really do not have a strong clue about what these patients really looked like and whether a patient I am 
seeing is going to be similar to what was reported." Thus, I think those are the real strengths of what you reported, and, certainly, the lack of any recurrence in those who underwent complete resection is pretty exciting.

My first question is about the choice of chemoradiotherapy versus chemotherapy. I did a brief review of the studies that have been published on induction chemotherapy, certainly patients who were not quite as well characterized but had stage III thymoma or thymic carcinoma, and I think that your complete resection rate was pretty similar, $77 \%$ versus $75 \%$. Your survival was pretty similar at 5 years, $71 \%$ versus $74 \%$. However, your CPR rate was lower, $0 \%$ versus $18 \%$ in the other studies, and the partial response rate was lower also, about $46 \%$ versus $89 \%$. So I am not necessarily seeing a benefit as I would have expected from chemoradiotherapy, and I am wondering about your thoughts about that.

Dr Robert J. Korst. Thank you, Dr Detterbeck.

We had the same impression as well. There have been a couple of CPRs in at least 1 of the other prospective clinical trials of this disease, but we did not see any, despite the addition of another locally acting agent. We did, however, have 5 near CPRs in the patients with thymic carcinoma. I think from a toxicity standpoint, we are in the ballpark with chemotherapy alone. I do not think our study had significantly more toxicity than some of the other published data. The studies, however, were drastically different in terms of the stages included and other inclusion criteria. The patients had been deemed unresectable in the other 2 prospective studies, but that is a highly subjective term. In addition to that, the chemotherapy agents were different. One of the studies was actually a chemotherapy and prednisone study. Thus, it is really difficult to make comparisons. What we tried to do with the present study was to try and make some of these factors as objective as possible, such that in the future, when other studies are done, the data can be compared.

It is hard to say I am going to change my practice because of a 22-patient prospective, single-arm trial, but it is all we have in thymoma. I have always treated with preoperative chemotherapy for locally advanced thymoma; however, the data for the thymic carcinoma are intriguing. Of the 7 patients, 4 had a near CPR. It is also the patients with thymic carcinoma who also had the most significant radiographic response. Therefore, if I had a patient with thymic carcinoma who presented to me and met these criteria, I would probably treat them with chemoradiotherapy up front.

Dr Detterbeck. My next question is where do we go from here? This is an intriguing trial, 20 patients. It is slightly more difficult to really know for sure whether it is chemoradiotherapy versus chemotherapy, et cetera, et cetera, what is really the way to go and how do we get more science behind what we should be doing as we move forward.

Dr Korst. Well, what we really need to do is band together and start doing trials in this rare disease. I can tell you that I shopped this trial around to anyone who would listen 5 years ago, and the response was always similar, which was, "We are going to see 2 patients this year. It is really not worth our while to get this through our institutional review board." We have to change that, and if we can change that, we can start investigating all types of questions for these patients.
Dr Scott J. Swanson (Boston, Mass). I really enjoyed it, Rob.

Without seeing the scans, it is hard for us to know what you were looking at. How comfortable are you that these patients had unresectable disease before treatment and is there some other minimally invasive method we should use to ensure that? Because if 4 or 5 of these patients had resectable disease before this treatment, perhaps they did not need this intensive therapy up front.

Dr Korst. Well, that is clearly a good point, and I will be the first to tell you that unresectability was not an inclusion criterion. There were some patients who, if you looked at the scan, you would say, "I could probably take that out," and then there actually were some patients who had terrible disease. The remainder of the patients were all kind of on that fence. One third of the patients had clear-cut great vessel or organ invasion, indicating an intraluminal tumor. It is hard to make comparisons in this disease because my "unresectable" might not be your "unresectable."

Dr Swanson. I have 1 last follow-up question. Carcinoma sounds clear and clear-cut invasion sounds clear. If you have a patient you are not so sure about now but who fits these criteria, would you give them the induction therapy or would you try to resect them, you know, off the protocol?

Dr Korst. If I was treating off the protocol, I think what I would do is probably treat with preoperative chemotherapy for patients with marginally resectable disease at this point, unless they were a patient with carcinoma or perhaps B3, for whom I would use chemoradiotherapy.

Dr Swanson. So nobody progresses. You do not lose the ability to resect anybody.

Dr Korst. No one progressed.

Dr Shaf Keshavjee (Toronto, Ontario, Canada). I have just a comment to Scott's question. When you consider the survival curves of thymoma and you consider the ones that we resect that recur later, that was sort of the target we were looking at, the high risk of recurrent tumors or a high risk of incomplete resection. So, the patients who you see clearly do not have resectable disease and "let us try and treat them and see whether we can make them resectable" were not really the target for this trial. It was more the high-risk larger tumors in general, but not necessarily just large, and then showing invasive or aggressive features, to see whether we could improve on that. The pleasant surprise was how well the thymic carcinomas behave with this induction therapy. So, those were 2 important nuances.

Dr Jacques-Pierre Fontaine (Tampa, Fla). Of the $23 \%$ of your patients who did not undergo an R0 resection, what percentage were able to receive postoperative radiotherapy for those positive margins, seeing that they all had already received 4000 to 4500 cGy preoperatively? As a follow-up comment, if your trial does not show an increased rate of R0 resectability or a change in survival compared with previous series, albeit not truly comparable, why not simply give them induction chemotherapy only, resect, and treat your R1 and R2 resections with higher doses of uninterrupted postoperative radiotherapy, which would be more effective than lower doses or interrupted doses of radiation?

Dr Korst. Of the patients who did not undergo an R0 resection, 1 underwent debulking and died postoperatively, but the other 4 were treated. 
Dr Fontaine. So, they were able to undergo radiotherapy despite the initial radiation?

Dr Korst. They were able to be treated. Again, it is a matter of philosophy. The purpose of the trial was to try to enhance our rate of complete resection by adding another local modality. It is unfortunate that the trials are really not comparable. The other trials excluded patients with thymic carcinoma. One third of our patients had carcinoma. You just cannot compare the patients.

Dr Dirk Van Raemdonck (Leuven, Belgium). With regard to your pretreatment staging, did you do thoracoscopy in any of these patients or in all patients to exclude stage IVA disease?

Dr Korst. No. It was all done radiographically. A single patient was found to have stage IVA disease once they had undergone resection. Also, we had 2 patients who had stage IVB because of nodal disease.

Dr Joshua R. Sonett (New York, NY). It was a great endeavor and great project, with results that I think will be extremely helpful. One question that I have encountered is trying to sort out the large thymomas that will not respond to induction therapy and, thus, should avoid induction. Thus, using pathology or molecular studies, we need to separate out who should receive therapy for thymoma. I have had a number of patients with very large thymomas that had very disappointing results from induction chemotherapy. I am wondering whether you could glean anything from your data that indicates which of those thymomas responded and which did not, because if they are not going to respond, clearly, the whole debate about whether to go straight to surgery or give them induction is less relevant.

Dr Korst. Most type A thymomas can be resected. I think the WHO histologic type plays a large role in the decision. However, the carcinoma or B3 cases, I think those are the ones for which one must entertain the idea of induction therapy. 
TABLE E1. Tumor details of 21 individual patients on the protocol

\begin{tabular}{|c|c|c|c|c|c|c|c|c|c|}
\hline Pt. ID* & $\begin{array}{c}\text { Tumor } \\
\text { size }(\mathbf{c m}) \dagger\end{array}$ & $\begin{array}{c}\text { Initial } \\
\mathbf{S U V}_{\max } \ddagger \\
\end{array}$ & CT criteria & $\begin{array}{c}\text { WHO histologic } \\
\text { type } \S\end{array}$ & $\begin{array}{c}\text { Change in } \\
\text { tumor size }(\%) \\
\end{array}$ & $\begin{array}{c}\text { Change in } \\
\operatorname{SUV}_{\max }(\%) \\
\end{array}$ & $\begin{array}{c}\text { Resection } \\
\text { status } \\
\end{array}$ & Stage $\llbracket$ & $\begin{array}{c}\text { Viable } \\
\text { tumor\# }(\%)\end{array}$ \\
\hline $715-01$ & 5 & 4.2 & $\begin{array}{l}\text { Lobulated } \\
\text { Heterogeneous }\end{array}$ & B3 & 0 & -52 & R0 & III & 10 \\
\hline $715-02$ & 8.3 & 5.4 & $\begin{array}{l}\text { Lobulated } \\
\text { BCV invasion }\end{array}$ & Metaplastic & -12 & -48 & R0 & II & 100 \\
\hline $715-03$ & 6.1 & 8.7 & $\begin{array}{l}\text { Lobulated } \\
\text { Heterogeneous } \\
\text { IA invasion }\end{array}$ & $\mathrm{C}$ & -25 & -28 & $\mathrm{R} 1$ & IVB & 80 \\
\hline $715-04$ & 8 & 5.1 & $\begin{array}{l}\text { Lobulated } \\
\text { Heterogeneous }\end{array}$ & B3 & -50 & -41 & R0 & III & 90 \\
\hline $715-05$ & 10.3 & 11.2 & $\begin{array}{l}\text { Lobulated } \\
\text { Heterogeneous } \\
\text { AA encirclement }\end{array}$ & B3 & -5 & -36 & $\mathrm{R} 2$ & III & 100 \\
\hline $715-06$ & 7.5 & 6.3 & $\begin{array}{l}\text { Calcified } \\
\text { Heterogeneous } \\
\text { SVC invasion }\end{array}$ & B3 & -39 & -49 & $\mathrm{R} 1$ & III & 100 \\
\hline $715-07$ & 7.2 & 3.9 & $\begin{array}{l}\text { Heterogeneous } \\
\text { Calcified }\end{array}$ & B2 & -8 & -10 & R0 & I & 100 \\
\hline $715-08$ & 14 & 2.9 & $\begin{array}{l}\text { Lobulated } \\
\text { Heterogeneous } \\
\text { Calcified }\end{array}$ & $\mathrm{AB}$ & -32 & -7 & R0 & III & 75 \\
\hline $715-09$ & 5.1 & 3.5 & $\begin{array}{l}\text { Lobulated } \\
\text { Heterogeneous }\end{array}$ & B2 & -2 & -100 & R0 & II & 95 \\
\hline $715-10$ & 7 & 3.7 & Lobulated & B2 & -43 & -100 & R0 & III & 80 \\
\hline $715-11$ & 5.6 & 4.6 & Lobulated & B3 & -30 & -25 & R0 & III & NR \\
\hline $715-12$ & 6.3 & 5.3 & Lobulated & $\mathrm{C}$ & -19 & -39 & R0 & III & 10 \\
\hline $715-13$ & 6.7 & 3.3 & $\begin{array}{l}\text { Lobulated } \\
\text { Heterogeneous }\end{array}$ & A & -3 & -39 & R0 & II & 85 \\
\hline $712-01$ & 5 & 10.1 & $\begin{array}{l}\text { Heterogeneous } \\
\text { Paralyzed diaphragm }\end{array}$ & $\mathrm{C}$ & -54 & -100 & R0 & III & 10 \\
\hline $712-02$ & 9 & 1.5 & Heterogeneous & A & -17 & +87 & R0 & III & 15 \\
\hline $712-03$ & 6.6 & 4.2 & $\begin{array}{l}\text { Lobulated } \\
\text { Heterogeneous }\end{array}$ & B1 & -36 & -100 & R0 & II & 40 \\
\hline 714-01 & 5.3 & NR & $\begin{array}{l}\text { Lobulated } \\
\text { Heterogeneous }\end{array}$ & $\mathrm{C}$ & -43 & NR & R0 & III & 95 \\
\hline $714-02$ & 6.6 & NR & $\begin{array}{l}\text { Lobulated } \\
\text { Heterogeneous } \\
\text { IA encirclement }\end{array}$ & $\mathrm{C}$ & -28 & NR & $\mathrm{R} 1$ & IVB & 10 \\
\hline $717-01$ & 7.3 & 15 & $\begin{array}{l}\text { Lobulated } \\
\text { Main PA invasion }\end{array}$ & $\mathrm{C}$ & -23 & -37 & R0 & III & 80 \\
\hline $717-02$ & 6.8 & 14 & SVC encirclement & $\mathrm{C}$ & -41 & -100 & R0 & I & 1 \\
\hline $717-03$ & 14 & 6.5 & $\begin{array}{l}\text { Lobulated } \\
\text { Heterogeneous } \\
\text { Calcified } \\
\text { RML invasion }\end{array}$ & B3 & -36 & -72 & R0 & IVA & 40 \\
\hline
\end{tabular}

Pt. ID, Patient identification; $S U V_{\max }$, maximum standard uptake value; $C T$, computed tomography; $W H O$, World Health Organization; $B C V$, brachiocephalic vein; $I A$, innominate artery; $A A$, ascending aorta; $S V C$, superior vena cava; $N R$, not reported; $P A$, pulmonary artery; $R M L$, right middle lobe of the lung. *University of Toronto, $\mathrm{n}=715$; The Valley

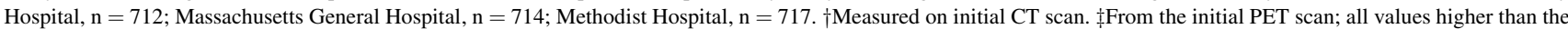
mediastinal blood pool. §From resected specimen. ||Measured on CT scan. ๆPostoperative Masaoka stage. \#Estimated by institutional pathologists. 